\title{
The Reeh-Schlieder Property for Quantum Fields on Stationary Spacetimes
}

\author{
Alexander Strohmaier \\ Universität Leipzig, Institut für theoretische Physik, Augustusplatz 10/11, D-04109 \\ Leipzig, Germany \\ E-mail: alexander.strohmaier@itp.uni-leipzig.de
}

\begin{abstract}
We show that as soon as a linear quantum field on a stationary spacetime satisfies a certain type of hyperbolic equation, the (quasifree) ground- and KMS-states with respect to the canonical time flow have the Reeh-Schlieder property. We also obtain an analog of Borchers' timelike tube theorem. The class of fields we consider contains the Dirac field, the Klein-Gordon field and the Proca field.
\end{abstract}

Mathematics Subject Classification (2000): 81T05, 81T20, 35J15, 35L10

\section{Introduction}

For the analysis of quantum field theory in curved spacetime it has turned out that the framework of algebraic quantum field theory (see [19]) is most suitable for analyzing the problems connected with the non-uniqueness of the quantization of linear fields (see e.g. [38] and the references therein). The problem reduces to finding appropriate representations of the field algebra which can straightforwardly be constructed on manifolds (see [13, 14]). This is the same as specifying 
vacuum-like states over the algebra. On stationary spacetimes it is possible to distinguish states as ground- or KMS-states with respect to the canonical time translations. For free fields satisfying certain wave-equations it was recently shown in [34] that such passive quasifree states satisfy the microlocal spectrum condition (see [8, 32]) which is believed to be a substitute for the usual spectrum condition in Minkowski spacetime. For the case of the scalar field on a 4-dimensional globally hyperbolic spacetime it was shown in [32] that the microlocal spectrum condition is equivalent to the requirement that the 2-point function is of Hadamard form (see e.g. [15, 38]), which allows for a renormalization of the stress energy tensor ([37]). It therefore seems reasonable to consider ground- or KMS-states for free quantum fields on stationary spacetimes as good substitutes for the vacuum in flat spacetime.

In the Minkowski space theory the vacuum vector turns out to be cyclic for field algebras associated to nonvoid open regions ([33]). This Reeh-Schlieder property of the vacuum vector holds also for thermal quantum field theories ([22]). By the lack of symmetry in general spacetimes it is not clear whether physically reasonable vacuum states have this property as well. It was shown in [36] that the quasifree ground-state of the massive scalar field on an ultrastatic spacetime is of this kind. The proof uses an anti-locality property of the square root of the Laplace operator. Using similar methods it was possible to obtain a Reeh-Schlieder-type property for solutions to the Dirac equation on an ultrastatic spacetime with compact Cauchy surface in [3], and the Reeh-Schlieder property for ground- and KMSstates of the free Dirac field on a static globally hyperbolic 4-dimensional spacetime in [35].

We will show in this paper that the Reeh-Schlieder property of ground- and KMSstates holds for a large class of free fields on stationary spacetimes. We introduce the notions of (classical) linear fermionic and bosonic field theories on a spacetime and show that these notions lead via canonical quantization to quantum field theories on this spacetime. We note that our approach does not use an initial data formulation. In the last section we show how the most common free fields fit into this framework. Our main theorem states that as soon as the classical field fulfills a certain hyperbolic partial differential equation, a state over the field algebra of the quantized theory, which is a (quasifree in the bosonic case) ground- or KMS-state with respect to the group of time translations, has the Reeh-Schlieder property. 
In order to show this we combine the Gelfand-Maurin theorem on generalized eigenvectors with classical results on the strong unique continuation property of solutions of certain second order elliptic differential equations. As a result we obtain a curved spacetime analog to the timelike tube theorem of Borchers ([6]). Using standard arguments this yields the Reeh-Schlieder property.

The class of fields which fulfill our assumptions contains the Dirac field, the Proca field and the scalar field on arbitrary connected 4-dimensional globally hyperbolic stationary Lorentzian manifolds. We note that as a consequence the HartleHawking state for the Klein-Gordon field on the external Schwarzschild spacetime has the Reeh-Schlieder property (see [25]). This is a new result which so far has not been obtained by the methods previously employed.

The Reeh-Schlieder property serves as the starting point for the use of the TomitaTakesaki-theory within quantum field theory. The application of this theory to the analysis of quantum field theory in Minkowski spacetime was very fruitful (see e.g. [5]) and there might be an impact as well on curved space quantum physics (see [9],[18]).

\section{Classical linear field theories}

In the following and throughout the text a smooth manifold will always be Hausdorff and separable as a topological space. If we are given a smooth vector bundle $E$ over a smooth manifold $M$ one can endow the space of smooth sections $\Gamma(E)$ and the space of compactly supported smooth sections $\Gamma_{0}(E)$ with locally convex topologies (see [11, 12]) in a similar way as for $C^{\infty}\left(\mathbb{R}^{n}\right)$ and $C_{0}^{\infty}\left(\mathbb{R}^{n}\right)$. These locally convex vector spaces turn out to be nuclear (see [29], or [31, 23] for properties of nuclear spaces). We denote the topological dual of the space $\Gamma_{0}(E)$ by $\mathcal{D}^{\prime}\left(M, E^{*}\right)$, calling it the space of distributions with values in the dual bundle $E^{*}$. For some open $\mathcal{O} \subset M$ the subspaces $\Gamma_{0}(E, \mathcal{O})$ and $\Gamma(E, \mathcal{O})$ of sections supported in $\mathcal{O}$ are closed. We call a smooth vector bundle $E$ a $G$-vector bundle for some Lie-group $G$ if there is a smooth action of $G$ on the base space $M$ and on $E$, such that the bundle projection $E \rightarrow M$ is equivariant and the left action $q: E_{x} \rightarrow E_{q x}$ is linear for all $q \in G$ and $x \in M$. In this case we have a canonical action of $G$ on $\Gamma_{0}(E)$ and $\Gamma(E)$, for which we use the notation $G \times \Gamma(E) \rightarrow \Gamma(E),(q, f) \rightarrow q f$. These representations of $G$ are continuous in 
the corresponding locally convex topologies. A smooth n-dimensional Lorentzian manifold $(M, g)$ is a smooth n-dimensional manifold with smooth metric $g$ of constant signature $(1,-1, \ldots,-1)$ and $n \geq 2$ (see e.g. [30]). For any open set $\mathcal{O}$ we denote the open causal complement of $\mathcal{O}$, i.e. the interior of the set of points in $M$ which cannot be joined to a point in $\mathcal{O}$ by a causal curve, by $\mathcal{O}^{\perp}$. If $E$ is a smooth vector bundle over a Lorentzian manifold $(M, g)$ we say a second order differential operator $P: \Gamma(E) \rightarrow \Gamma(E)$ has metric principal part, if in local coordinates the principal part is of the form $1 \cdot g^{i k} \partial_{i} \partial_{k}$.

\subsection{The fermionic case}

Let $(M, g)$ be a smooth n-dimensional Lorentzian manifold. We denote the identity component of the group of isometries of $M$ by $G$ and its universal covering group by $\tilde{G}$. Note that $\tilde{G}$ is a Lie-group which acts smoothly on $M$.

Definition 2.1. A linear fermionic field theory on $M$ is a 5-tuple $(\mathcal{H}, \mathcal{K}, E, \eta, \rho)$, where $\mathcal{H}$ is a complex Hilbert space with conjugation $\mathcal{K}, E$ a smooth complex $\tilde{G}$ vector bundle, $\eta$ a linear map $\eta: \Gamma_{0}(E) \rightarrow \mathcal{H}$ with dense range, and $\rho$ a unitary representation of $\tilde{G}$ on $\mathcal{H}$ commuting with $\mathcal{K}$, such that the following conditions are satisfied:

1. Covariance: $\eta(q f)=\rho(q)(\eta(f)), \quad \forall f \in \Gamma_{0}(E), q \in \tilde{G}$.

2. Causality: $\mathcal{O}_{1} \subset \mathcal{O}_{2}^{\perp}$ implies $\mathcal{H}\left(\mathcal{O}_{1}\right) \perp \mathcal{H}\left(\mathcal{O}_{2}\right)$, where $\mathcal{H}(\mathcal{O})$ denotes the closure of the image of $\Gamma_{0}(E, \mathcal{O})$ under $\eta$.

3. Continuity: $\eta$ is weakly continuous, i.e. $\langle v, \eta(\cdot)\rangle$ is in $\mathcal{D}^{\prime}\left(M, E^{*}\right)$ for all $v \in \mathcal{H}$.

4. $\mathcal{K}$ is local, i.e. $\mathcal{K}(\mathcal{H}(\mathcal{O}))=\mathcal{H}(\mathcal{O})$.

Proposition 2.2. For any linear fermionic field theory $(\mathcal{H}, \mathcal{K}, E, \eta, \rho)$ on $M$ the map $\eta$ is norm continuous. Moreover, the representation $\rho$ is strongly continuous.

Proof. The sesquilinear form $B(f, g):=\langle\eta(f), \eta(g)\rangle$ on $\Gamma_{0}(E) \times \Gamma_{0}(E)$ is separately continuous, and as a consequence of the nuclearity of $\Gamma_{0}(E)$ it is jointly continuous. This gives the norm continuity of $\eta$. Since the action of $\tilde{G}$ on $E$ is 
smooth, the action of $\tilde{G}$ on $\Gamma_{0}(E)$ is continuous. As a consequence we obtain the strong continuity of $\rho$.

\subsection{The bosonic case}

Let $(M, g)$ be again a smooth n-dimensional Lorentzian manifold and $G$ be the identity component of the group of isometries of $M$.

Definition 2.3. A linear bosonic field theory on $M$ is a 5-tuple ( $\mathcal{W}, \sigma, E, \eta, \rho)$, where $\mathcal{W}$ is a symplectic vector space with symplectic form $\sigma, E$ a smooth real $G$-vector bundle, $\eta$ a surjective linear map $\eta: \Gamma_{0}(E) \rightarrow \mathcal{W}$, and $\rho$ a representation of $G$ on $\mathcal{W}$ by symplectomorphisms, such that the following conditions are satisfied:

1. Covariance: $\eta(q f)=\rho(q)(\eta(f)), \quad \forall f \in \Gamma_{0}(E), q \in G$.

2. Causality: $\mathcal{O}_{1} \subset \mathcal{O}_{2}^{\perp}$ implies $\mathcal{W}\left(\mathcal{O}_{1}\right) \perp \mathcal{W}\left(\mathcal{O}_{2}\right)$, where $\mathcal{W}(\mathcal{O})$ denotes the image of $\Gamma_{0}(E, \mathcal{O})$ under $\eta$.

3. Continuity: For all $v \in \mathcal{W}$ the map $\Gamma_{0}(E) \rightarrow \mathbb{R}, f \rightarrow \sigma(v, \eta(f))$ is continuous, i.e. defines a distribution in $\mathcal{D}^{\prime}\left(M, E^{*}\right)$.

\section{Canonical Quantization}

In this section $(M, g)$ will be a smooth n-dimensional Lorentzian manifold. We will work in the framework of algebraic quantum field theory ([19]). A quantum field theory on $M$ will be defined by a net of local field algebras, i.e. a map $\mathcal{O} \rightarrow \mathcal{F}(\mathcal{O})$ from the relatively compact open subsets of $M$ to the set of closed *-subalgebras of a $C^{*}$ - or $W^{*}$-algebra $\mathcal{F}$ which is isotone, i.e. $\mathcal{F}\left(\mathcal{O}_{1}\right) \subset \mathcal{F}\left(\mathcal{O}_{2}\right)$ whenever $\mathcal{O}_{1} \subset \mathcal{O}_{2}$.

\subsection{Quantization of a linear fermionic field theory}

We show how a linear fermionic field theory gives rise to a quantum field theory on $M$ given by a net of local field algebras.

Given a linear fermionic field theory $(\mathcal{H}, \mathcal{K}, E, \eta, \rho)$, the field algebra $\mathcal{F}$ is the 
(self-dual) CAR-algebra $\operatorname{CAR}(\mathcal{H}, \mathcal{K})$ (see [1] $)$. This is the $C^{*}$-algebra with unit generated by symbols $B(v)$ with $v \in \mathcal{H}$ and the relations

$$
\begin{gathered}
v \rightarrow B(v) \quad \text { is complex linear, } \\
B(v)^{*}=B(\mathcal{K} v), \\
\left\{B\left(v_{1}\right), B\left(v_{2}\right)\right\}=B\left(v_{1}\right) B\left(v_{2}\right)+B\left(v_{2}\right) B\left(v_{1}\right)=\left\langle\mathcal{K} v_{1}, v_{2}\right\rangle .
\end{gathered}
$$

$\mathcal{F}$ has a natural $\mathbb{Z}_{2}$-grading. The even/odd parts are spanned by those products $B\left(v_{1}\right) \ldots B\left(v_{k}\right)$ with an even/odd number of generators. For each relatively compact subset $\mathcal{O} \subset M$ we define the local algebra $\mathcal{F}(\mathcal{O}) \subset \mathcal{F}$ to be the closed unital *-subalgebra generated by the symbols $B(\eta(f))$ with $f \in \Gamma_{0}(E, \mathcal{O})$. The representation $\rho$ of the group $\tilde{G}$ on $\mathcal{H}$ gives rise to a representation $\tau$ of $\tilde{G}$ by strongly continuous Bogoliubov automorphisms of the algebra (see [[1]). It is not difficult to check the following properties of the net $\mathcal{O} \rightarrow \mathcal{F}(\mathcal{O})$ :

1. Isotony: $\mathcal{O}_{1} \subset \mathcal{O}_{2}$ implies $\mathcal{F}\left(\mathcal{O}_{1}\right) \subset \mathcal{F}\left(\mathcal{O}_{2}\right)$.

2. Causality: if $\mathcal{O}_{1} \subset \mathcal{O}_{2}^{\perp}$, then $\left\{\mathcal{F}\left(\mathcal{O}_{1}\right), \mathcal{F}\left(\mathcal{O}_{2}\right)\right\}=\{0\}$, where $\{\cdot, \cdot\}$ denotes the graded commutator.

3. Covariance: $\tau(q) \mathcal{F}(\mathcal{O})=\mathcal{F}(q \mathcal{O}) \quad \forall q \in \tilde{G}$.

Moreover, $\mathcal{F}$ is the quasilocal algebra of the net $\mathcal{O} \rightarrow \mathcal{F}(\mathcal{O})$ (see [7], proposition 5.2.6). Hence, this defines a reasonable quantum field theory on the manifold $M$. Note that $\mathcal{F}$ is not the algebra of observables. The algebra of observables $\mathcal{A}$ should be a ${ }^{*}$-subalgebra of $\mathcal{F}_{\text {even }}$ consisting of elements $a$ for which $\tau_{g_{1}} a=\tau_{g_{2}} a$ whenever $p\left(g_{1}\right)=p\left(g_{2}\right)$, where $p: \tilde{G} \rightarrow G$ is the covering map. Usually $\mathcal{A}$ is the $*$-subalgebra of elements which are invariant under the action of a gauge group.

\subsection{Quantization of a linear bosonic field theory}

Each linear bosonic field theory $(\mathcal{W}, \sigma, E, \eta, \rho)$ gives rise to a quantum field theory on $M$. The field algebra $\mathcal{F}$ is defined to be the $\operatorname{CCR}$-algebra $\operatorname{CCR}(\mathcal{W}, \sigma)$ (see [26, 27, 7]). This is the $C^{*}$-algebra generated by symbols $W(v)$ with $v \in \mathcal{W}$ and the relations

$$
\begin{gathered}
W(-v)=W(v)^{*} \\
W\left(v_{1}\right) W\left(v_{2}\right)=e^{-i \sigma\left(v_{1}, v_{2}\right) / 2} W\left(v_{1}+v_{2}\right) .
\end{gathered}
$$


We define for each relatively compact open subset $\mathcal{O} \subset M$ the local field algebra $\mathcal{F}(\mathcal{O}) \subset \mathcal{F}$ to be the closed $*$-subalgebra generated by the symbols $W(v)$ with $v \in \mathcal{W}(\mathcal{O})$.

The representation $\rho$ of $G$ gives rise to a representation $\tau$ of $G$ by Bogoliubov automorphisms of the algebra $\mathcal{F}$ (see e.g.[7]), and the net $\mathcal{O} \rightarrow \mathcal{F}(\mathcal{O})$ has the following properties:

1. Isotony: $\mathcal{O}_{1} \subset \mathcal{O}_{2}$ implies $\mathcal{F}\left(\mathcal{O}_{1}\right) \subset \mathcal{F}\left(\mathcal{O}_{2}\right)$.

2. Causality: if $\mathcal{O}_{1} \subset \mathcal{O}_{2}^{\perp}$, then $\left[\mathcal{F}\left(\mathcal{O}_{1}\right), \mathcal{F}\left(\mathcal{O}_{2}\right)\right]=\{0\}$.

3. Covariance: $\tau(q) \mathcal{F}(\mathcal{O})=\mathcal{F}(q \mathcal{O}) \quad \forall q \in G$.

Moreover, $\mathcal{F}$ is the quasilocal algebra of the net $\mathcal{O} \rightarrow \mathcal{F}(\mathcal{O})$ (see [7], proposition 5.2.10). Hence, this defines a reasonable quantum field theory on the manifold $M$. Unlike the fermionic case the representation $\tau$ fails to be strongly continuous whenever it is nontrivial. We therefore need to pass to certain representations of the field algebra to obtain a net of von Neumann algebras on which $\tau$ extends to a $\sigma$-weakly-continuous representation. In order to avoid complications we specialize to the so-called quasifree states. Assume that we are given a scalar product $\mu$ on $\mathcal{W}$ which dominates $\sigma$, i.e. satisfies the estimate

$$
\left|\sigma\left(v_{1}, v_{2}\right)\right|^{2} \leq 4 \mu\left(v_{1}, v_{1}\right) \mu\left(v_{2}, v_{2}\right) \quad v_{1}, v_{2} \in \mathcal{W}
$$

In this case the linear functional $\omega_{\mu}: \mathcal{F} \rightarrow \mathbb{C}$, defined by

$$
\omega_{\mu}(W(v)):=e^{-\mu(v, v) / 2} \quad v \in \mathcal{W}
$$

is a state (see [26, 27, 7]). The states over $\mathcal{F}$ which can be realized in this way are called quasifree states. A quasifree state $\omega_{\mu}$ gives rise to a one particle structure (Proposition 3.1 in [27]), that is a map $K_{\mu}: \mathcal{W} \rightarrow H_{\mu}$ to some complex Hilbert space $H_{\mu}$, such that

1. the complexified range of $K_{\mu}$, (i.e. $K_{\mu} \mathcal{W}+i K_{\mu} \mathcal{W}$ ), is dense in $H_{\mu}$,

2. $\left\langle K_{\mu} v_{1}, K_{\mu} v_{2}\right\rangle=\mu\left(v_{1}, v_{2}\right)+\frac{i}{2} \sigma\left(v_{1}, v_{2}\right)$. 
This structure is unique up to equivalence. A one particle structure $\left(K_{\mu}, H_{\mu}\right)$ for a quasifree state allows one to construct the GNS-triple $\left(\pi_{\omega_{\mu}}, \mathcal{H}_{\omega_{\mu}}, \Omega_{\omega_{\mu}}\right)$ explicitly (see [27, 26, 7]). Namely, one takes $\mathcal{H}_{\omega_{\mu}}$ to be the bosonic Fock space over $H_{\mu}$ with Fock vacuum $\Omega_{\omega_{\mu}}$, and defines $\pi_{\omega_{\mu}}(W(v))=\exp \left(-\left(\overline{\hat{a}^{*}(K v)-\hat{a}(K v)}\right)\right)$, where $\hat{a}^{*}(\cdot)$ and $\hat{a}(\cdot)$ are the usual creation and annihilation operators. One clearly has the following

Proposition 3.1. Let $\omega_{\mu}$ be a quasifree state over the CCR-algebra $\mathcal{F}=C C R(\mathcal{W}, \sigma)$ and let $\left(\pi_{\omega_{\mu}}, \mathcal{H}_{\omega_{\mu}}, \Omega_{\omega_{\mu}}\right)$ be its GNS-triple. If $V \subset \mathcal{W}$ is a subspace which is dense in $\mathcal{W}$ in the topology defined by $\mu$, then the *-algebra generated by the set

$$
\left\{\pi_{\omega_{\mu}}(W(v)), v \in V\right\} \subset \pi_{\omega_{\mu}}(\mathcal{F})
$$

is strongly dense in the von Neumann algebra $\pi_{\omega_{\mu}}(\mathcal{F})^{\prime \prime}$.

Definition 3.2. Let $\mathcal{F}$ be a field algebra constructed from a linear bosonic field theory $(\mathcal{W}, \sigma, E, \eta, \rho)$ on $M$. We call a quasifree state $\omega_{\mu}$ over $\mathcal{F}$ continuous if the map

$$
\Gamma_{0}(E) \times \Gamma_{0}(E) \rightarrow \mathbb{R}, \quad\left(f_{1}, f_{2}\right) \rightarrow \mu\left(\eta\left(f_{1}\right), \eta\left(f_{2}\right)\right)
$$

is continuous and hence defines a distribution in $\mathcal{D}^{\prime}\left(M \times M, E^{*} \otimes E^{*}\right)$, where $E^{*} \otimes E^{*}$ is the direct product of the bundle $E^{*}$ with itself over the base space $M \times M$.

This is clearly a necessary and sufficient condition for Wightman 2-point function $w_{2}(\cdot, \cdot):=\langle K \eta(\cdot), K \eta(\cdot)\rangle$ to be a distribution. For the class of continuous quasifree states we can circumvent the problems connected with the noncontinuity of the representation $\tau$ of $G$ on $\mathcal{F}$. Since the action of $G$ is continuous on $\Gamma_{0}(E)$ and $\rho$ leaves $\sigma$ and $\mu$ invariant, there exists a unique strongly continuous representation $\tilde{U}$ of $G$ on the one particle Hilbert space $H_{\mu}$, such that $K_{\mu} \circ \rho(q)=$ $\tilde{U}(q) \circ K_{\mu}$ for all $q \in G$. Second quantization gives a strongly continuous unitary representation $U$ of $G$ on $\mathcal{H}_{\omega_{\mu}}$, such that $\pi_{\omega_{\mu}}(\rho(q) a)=U(q) \pi_{\omega_{\mu}}(a) U^{-1}(q)$ for all $q \in G$ and $a \in \mathcal{F}$. Hence, one gets the following proposition:

Proposition 3.3. Let $\omega_{\mu}$ be a continuous $G$-invariant quasifree state over the field algebra $\mathcal{F}$ constructed from a linear bosonic field theory $(\mathcal{W}, \sigma, E, \eta, \rho)$ on $M$. 
Let $\left(\pi_{\omega_{\mu}}, \mathcal{H}_{\omega_{\mu}}, \Omega_{\omega_{\mu}}\right)$ be its GNS-triple and $U$ be the unitary representation of $G$ on $\mathcal{H}_{\omega_{\mu}}$ induced by $\tau$. Then $U$ is strongly continuous and hence $\tau$ can be continued to a $\sigma$-weakly-continuous representation $\hat{\tau}$ of $G$ by automorphisms of the von Neumann algebra $\hat{\mathcal{F}}:=\pi_{\omega_{\mu}}(\mathcal{F})^{\prime \prime}$.

Therefore, given a continuous quasifree state $\omega_{\mu}$ we can construct the net of von Neumann algebras $\mathcal{O} \rightarrow \hat{\mathcal{F}}(\mathcal{O}):=\pi_{\omega_{\mu}}(\mathcal{F}(\mathcal{O}))^{\prime \prime}$. This assignment is isotone, causal and covariant, and the representation $\hat{\tau}$ of $G$ is $\sigma$-weakly-continuous. It gives rise to a quantum field theory on $M$ with reasonable physical properties.

\section{The Reeh-Schlieder property for quantized linear fields}

\subsection{Stationary spacetimes}

Let $(M, g)$ be an $\mathrm{n}$-dimensional time-oriented Lorentzian manifold which admits a one parameter group $h_{t}$ of isometries, smooth in $t$, with timelike orbits giving rise to a timelike Killing vector field $\xi$. Such a manifold is called stationary. For later considerations we need a special class of charts.

Lemma 4.1. For each point $p \in M$ there exists an open neighbourhood $\mathcal{O}$ and a chart $\phi: \mathcal{O} \rightarrow \mathbb{R}^{n}$ with coordinates $\left(x_{0}, \ldots, x_{n-1}\right)$, such that in local coordinates

1. $\xi=\frac{\partial}{\partial x_{0}}$,

2. the $(n-1) \times(n-1)$ matrix $-g^{\alpha \beta}(x), \alpha, \beta=1, \ldots, n-1$ is positive for all $x \in \phi(\mathcal{O})$.

Proof. One can always choose a neighbourhood $\mathcal{O}_{1}$ of $p$ and a chart $\phi: \mathcal{O}_{1} \rightarrow \mathbb{R}^{n}$ with coordinates $\left(x_{0}, \ldots, x_{n-1}\right)$, such that $\xi=\frac{\partial}{\partial x_{0}}$ and the dual metric tensor is diagonal in the point $p$, i.e.

$$
-g^{i k}(\phi(p))=\operatorname{diag}_{n}(g(\xi, \xi),-1, \ldots,-1) .
$$

$-g^{\alpha \beta}(\phi(p)), \alpha, \beta=1, \ldots, n-1$ is then a positive matrix. Since the matrixvalued function $-g^{\alpha \beta}$ is continuous, there exists a neighbourhood $\phi(\mathcal{O})$ of $\phi(p)$, on which it is positive. 


\subsection{Free quantum fields on stationary spacetimes}

Since $h_{t}$ is a group of isometries it defines a group homomorphism $\mathbb{R} \rightarrow G$ which lifts uniquely to a group homomorphism $\mathbb{R} \rightarrow \tilde{G}$. Hence, in case we have a net of field algebra $\mathcal{O} \rightarrow \mathcal{F}(\mathcal{O})$ constructed from a linear fermionic or bosonic field theory we canonically get a one parameter group of automorphisms $\tau_{t}$ which acts covariantly, i.e. $\tau_{t} \mathcal{F}(\mathcal{O})=\mathcal{F}\left(h_{t} \mathcal{O}\right)$. We call this group the group of canonical time translations induced by $h_{t}$. In the interesting cases one can realize $\mathcal{F}$ on a Hilbert space, such that $\tau_{t}$ is $\sigma$-weakly-continuous and hence extends to an automorphism group $\hat{\tau}_{t}$ of the von Neumann algebra $\mathcal{F}^{\prime \prime}$ (see e.g. proposition 3.3). It is then possible to distinguish vacuum states over the field algebra $\mathcal{F}$ as groundor KMS-states with respect to the group of time translations $\tau_{t}$.

Definition 4.2. Let $\mathcal{A}$ be a $W^{*}$-algebra and $\alpha_{t}$ be a $\sigma$-weakly-continuous oneparameter group of $*$-automorphisms of $\mathcal{A}$. An $\alpha_{t}$ invariant normal state $\omega$ is called ground-state with respect to $\alpha_{t}$ if the generator of the corresponding strongly continuous unitary group $U(t)$ on the GNS-Hilbert space is positive.

Definition 4.3. Let $\mathcal{A}$ and $\alpha_{t}$ be as above. A normal state $\omega$ is called KMS-state with inverse temperature $\beta>0$ with respect to $\alpha_{t}$ if for any pair $A, B \in \mathcal{A}$ there exists a complex function $F_{A, B}$ which is analytic in the strip

$$
\mathcal{D}_{\beta}:=\{z \in \mathbb{C} ; 0<\operatorname{Im}(z)<\beta\}
$$

and bounded and continuous on $\overline{\mathcal{D}_{\beta}}$, such that

$$
\begin{gathered}
F_{A, B}(t)=\omega\left(A \alpha_{t}(B)\right), \\
F_{A, B}(t+i \beta)=\omega\left(\alpha_{t}(B) A\right) .
\end{gathered}
$$

Definition 4.4. Let $\left(M, g, h_{t}\right)$ be a connected stationary Lorentzian manifold. Let $\{\mathcal{F}(\mathcal{O})\}$ be the net of local field algebras constructed from a linear fermionic field theory $(\mathcal{H}, \mathcal{K}, E, \eta, \rho)$ or from a linear bosonic field theory $(\mathcal{W}, \sigma, E, \eta, \rho)$. Denote the group of canonical time translations by $\tau_{t}$. Let $\omega$ be a $\tau_{t}$-invariant state over the quasilocal algebra $\mathcal{F}$, which we assume to be quasifree and continuous in the bosonic case, and denote by $\left(\pi_{\omega}, \mathcal{H}_{\omega}, \Omega_{\omega}\right)$ the corresponding GNS-triple. We say that $\omega$ is a ground or KMS state, if the unique normal extension of $\omega$ over $\pi_{\omega}(\mathcal{F})^{\prime \prime}$ is a ground or KMS state with respect to the unique $\sigma$-weakly-continuous extension of the group of time translations $\tau_{t}$. 
In the fermionic case the existence of ground states is always guaranteed ([1]]). Moreover, there exists a unique quasifree KMS-state with inverse temperature $\beta>0$ (see [7, 1]). In the bosonic case the construction of continuous quasifree ground- and KMS-states seems problematic in the general case. See e.g. [24] for conditions that allow the construction of a continuous quasifree ground state for the Klein-Gordon quantum field on a 4-dimensional stationary spacetime.

\subsection{A density theorem and a tube theorem}

Theorem 4.5. Let $\left(M, g, h_{t}\right)$ be a connected stationary Lorentzian manifold and E a smooth complex $h_{t}$-vector bundle. Let $H$ be a complex Hilbert space and $\rho_{t}$ a strongly continuous unitary one-parameter group on $H$. Assume that we have a linear strongly continuous map $\hat{\eta}: \Gamma_{0}(E) \rightarrow H$ with dense range which is covariant, i.e. $\hat{\eta}\left(h_{t} f\right)=\rho_{t} \hat{\eta}(f)$ for all $t \in \mathbb{R}, f \in \Gamma_{0}(E)$. Assume furthermore that $\hat{\eta} \circ P=0$ for some second order differential operator $P$ with metric principal part. Then $\hat{\eta}\left(\Gamma_{0}\left(E, h_{\mathbb{R}} \mathcal{O}\right)\right)$ is dense in $H$ for each nonvoid open set $\mathcal{O} \subset M$.

Proof. We introduce the following notations:

$$
\begin{gathered}
V:=\hat{\eta}\left(\Gamma_{0}\left(E, h_{\mathbb{R}} \mathcal{O}\right)^{\perp},\right. \\
p_{V} \ldots \text { orthogonal projection onto } V, \\
\Phi:=\operatorname{Ran}\left(p_{V} \circ \hat{\eta}\right) .
\end{gathered}
$$

$V$ is clearly a $\rho_{t}$-invariant subspace and $\Phi$ is dense in $V$. Identifying $\Phi$ with $\Gamma_{0}(E) / \operatorname{ker}\left(p_{V} \circ \hat{\eta}\right)$ we can endow $\Phi$ with the locally convex quotient topology. Since $\Gamma_{0}(E)$ is nuclear and $p_{V} \circ \hat{\eta}$ is continuous, $\Phi$ is a nuclear space (see [29, 31, 23]), and clearly the inclusion map $\Phi \rightarrow V$ is continuous. We denote the dual of $\Phi$ by $\Phi^{\prime}$. It follows that

$$
\Phi \subset V \subset \Phi^{\prime}
$$

is a Gelfand triple. We denote the selfadjoint generator of the group $\left.\rho_{t}\right|_{V}$ by $A$. Clearly, $\Phi \subset \mathcal{D}(A)$ and $A$ restricts to a continuous operator $\Phi \rightarrow \Phi$. Moreover $\Phi$ is invariant under the action of $\left.\rho_{t}\right|_{V}$. As a consequence $A$ is essentially selfadjoint on $\Phi$. Hence, there exists a complete set of generalized eigenvectors (see [17, 29]) 
for $A$, i.e. a family $v_{\lambda} \in \Phi^{\prime}$ indexed by a subset $I \subset \mathbb{R}$, such that

$$
\begin{gathered}
v_{\lambda}(A f)=\lambda v_{\lambda}(f), \quad \forall f \in \Phi, \\
v_{\lambda}(f)=0 \text { for all } \lambda \in I \Leftrightarrow f=0 .
\end{gathered}
$$

By continuity

$$
\psi_{\lambda}(\cdot):=v_{\lambda}\left(p_{V} \circ \hat{\eta}(\cdot)\right)
$$

defines for each $\lambda \in I$ a distribution in $\mathcal{D}^{\prime}\left(M, E^{*}\right)$, such that

$$
\begin{gathered}
\psi_{\lambda}\left(\Gamma_{0}\left(E, h_{\mathbb{R}} \mathcal{O}\right)\right)=\{0\}, \\
\psi_{\lambda}\left(\mathcal{L}_{\xi} f\right)=i \lambda \psi_{\lambda}(f), \\
\psi_{\lambda}(P \cdot)=0,
\end{gathered}
$$

where $\xi$ is the timelike Killing vector field induced by $h_{t}$ and $\mathcal{L}_{\xi}$ the Lie derivative on $\Gamma_{0}(E)$ defined by $\mathcal{L}_{\xi} f=\lim _{t \rightarrow 0} \frac{h_{t} f-f}{t}$.

For each point $p \in M$ there exists an open contractible neighbourhood $\mathcal{U}$ and a chart mapping $\mathcal{U}$ to $\mathbb{R}^{n}$ which we can choose to be of the form constructed in lemma 4.1. The restriction of $E$ to $\mathcal{U}$ is trivial and we can identify $\Gamma_{0}(E, \mathcal{U})$ with $C^{\infty}(\mathcal{U}) \otimes \mathbb{C}^{N}$ in such a way that $\mathcal{L}_{\xi} f=\frac{\partial}{\partial x_{0}} f$ for both functions and sections. We consider the distribution $\psi_{\lambda}$ in such a chart.

We have $P^{*} \psi_{\lambda}=0$, where $P^{*}$ is the adjoint operator. Moreover, equation (16) reads $\frac{\partial}{\partial x_{0}} \psi_{\lambda}=-i \lambda \psi_{\lambda}$. Note that the principal part of $P^{*}$ has the form

$$
\begin{gathered}
g^{00} \frac{\partial^{2}}{\partial x_{0}^{2}}+g^{0 \alpha} \frac{\partial^{2}}{\partial x_{0} \partial x_{\alpha}}+g^{\alpha \beta} \frac{\partial^{2}}{\partial x_{\alpha} \partial x_{\beta}} \\
\alpha, \beta=1,2, \ldots, n-1 .
\end{gathered}
$$

Replacing the $x_{0}$-derivatives by $-i \lambda$ and adding the term $-\frac{\partial^{2}}{\partial x_{0}^{2}}-\lambda^{2}$ we obtain an elliptic second order differential operator $P_{e}$ with $P_{e} \psi_{\lambda}=0$. Hence, $\psi_{\lambda}$ is smooth (see e.g. [21, 10]) and since $P_{e}$ has scalar principal part the classical result of Arozajn [2] (see especially Remark 3) Đimplies that $\psi_{\lambda}=0$ in each such chart in which $\psi_{\lambda}$ vanishes in an open nonvoid set, in particular in each such chart intersecting with $h_{\mathbb{R}} \mathcal{O}$. Since $M$ is connected this implies $\psi_{\lambda}=0$ on $M$. The set of generalized eigenvectors $v_{\lambda}$ was complete and therefore $V$ equals $\{0\}$ and the theorem is proved.

\footnotetext{
${ }^{1}$ For a detailed treatment see section 17.1 of [20] and the references therein
} 
As a consequence one gets a result similar to the timelike tube theorem ([6]) in Minkowski spacetime.

Theorem 4.6. Let $\left(M, g, h_{t}\right)$ be a connected stationary Lorentzian manifold. Let $\{\mathcal{F}(\mathcal{O})\}$ be a net of local field algebras constructed from a linear fermionic field theory $(\mathcal{H}, \mathcal{K}, E, \eta, \rho)$ or from a linear bosonic field theory $(\mathcal{W}, \sigma, E, \eta, \rho)$. Assume that $\eta \circ P=0$ for some second order differential operator $P$ with metric principal part. One has

1. The fermionic case. The *-subalgebra of $\mathcal{F}$ generated by the subset $\bigcup_{t \in \mathbb{R}} \mathcal{F}\left(h_{t} \mathcal{O}\right)$ is norm dense in $\mathcal{F}$ for each nonvoid relatively compact open set $\mathcal{O} \subset M$.

2. The bosonic case Let $\omega_{\mu}$ be a quasifree and continuous state over the quasilocal algebra $\mathcal{F}$. Assume that $\omega_{\mu}$ is invariant under the automorphism group $\tau_{t}$ induced by the Killing flow $h_{t}$. Denote its GNS-triple by $\left(\pi_{\omega_{\mu}}, \mathcal{H}_{\omega_{\mu}}, \Omega_{\omega_{\mu}}\right)$. The *-subalgebra of $\pi_{\omega_{\mu}}(\mathcal{F})$ generated by the subset $\bigcup_{t \in \mathbb{R}} \pi_{\omega_{\mu}}\left(\mathcal{F}\left(h_{t} \mathcal{O}\right)\right)$ is strongly dense in $\pi_{\omega_{\mu}}(\mathcal{F})$ for each nonvoid relatively compact open set $\mathcal{O} \subset M$.

Proof. We start with the fermionic case. Let $\mathcal{H}_{1}$ be the subspace of $\mathcal{H}$ generated by the set

$$
\left\{\eta\left(h_{t} f\right) \in \mathcal{H} ; f \in \Gamma_{0}(E, \mathcal{O}), t \in \mathbb{R}\right\}
$$

and let $\mathcal{F}_{1}$ be the unital $*$-subalgebra of $\mathcal{F}$ generated by the set $\left\{B(v), v \in \mathcal{H}_{1}\right\}$. Clearly, $\mathcal{F}_{1}$ is equal to the $*$-subalgebra of $\mathcal{F}$ generated by $\bigcup_{t \in \mathbb{R}} \mathcal{F}\left(h_{t} \mathcal{O}\right)$. The group of time translations $\rho_{t}$ is a strongly continuous one parameter group on $\mathcal{H}$, and with $\hat{\eta}=\eta$ we can apply theorem 4.5 above. It follows that $\mathcal{H}_{1}$ is dense in $\mathcal{H}$ and hence $\mathcal{F}_{1}$ is norm dense in $\mathcal{F}$.

In the bosonic case let $\mathcal{W}_{\mathbb{C}}$ be the complexification of $\mathcal{W}$ and take the complexification of $\mu$ as a scalar product on $\mathcal{W}_{\mathbb{C}}$. We complete this space and obtain a Hilbert space $H$. We complexify the real vector bundle $E$ and obtain the complex vector bundle $E_{\mathbb{C}} \cong E \oplus E$ with a canonical action of $h_{t}$. We can extend the map $\eta$ to a map $\eta_{\mathbb{C}}$ which maps from the section of $E_{\mathbb{C}}$ to $H$. By construction $\eta_{\mathbb{C}}$ has dense range. Since $\mu$ is invariant under the action of the time translations $\rho_{t}$ we get a strongly continuous unitary action of the group of time translations on $H$ 
such that $\eta_{\mathbb{C}}$ is equivariant. Let $H_{1}$ be the complex subspace of $H$ generated by the set

$$
\left\{\eta_{\mathbb{C}}\left(h_{t} f\right) \in H ; f \in \Gamma_{0}\left(E_{\mathbb{C}}, \mathcal{O}\right), t \in \mathbb{R}\right\}
$$

We see that all the assumptions for theorem 4.5 are fulfilled and hence $H_{1}$ is dense in $H$. Using proposition 3.1 one therefore concludes that the $*$-subalgebra of $\pi_{\omega_{\mu}}(\mathcal{F})$ generated by the set $\left\{W\left(\eta\left(h_{t} f\right)\right), f \in \Gamma_{0}(E, \mathcal{O}), t \in \mathbb{R}\right\}$ is strongly dense in $\pi_{\omega_{\mu}}(\mathcal{F})$.

\subsection{The Reeh-Schlieder property for ground- and KMS-states}

Definition 4.7. Let $\{\mathcal{F}(\mathcal{O})\}$ be a net of local field algebras indexed by the relatively compact open subsets of a manifold $M$. Let $\omega$ be a state over the quasilocal field algebra $\mathcal{F}$ and $\left(\pi_{\omega}, \mathcal{H}_{\omega}, \Omega_{\omega}\right)$ its GNS-triple. We say that $\omega$ has the ReehSchlieder property if $\Omega_{\omega}$ is cyclic for the von Neumann algebra $\pi_{\omega}(\mathcal{F}(\mathcal{O}))^{\prime \prime}$ for each nonvoid relatively compact open set $\mathcal{O} \subset M$.

Our main theorem is:

Theorem 4.8. Let $\left(M, g, h_{t}\right)$ be a connected stationary Lorentzian manifold. Let $\{\mathcal{F}(\mathcal{O})\}$ be the net of local field algebras constructed from a linear fermionic field theory $(\mathcal{H}, \mathcal{K}, E, \eta, \rho)$ or from a linear bosonic field theory $(\mathcal{W}, \sigma, E, \eta, \rho)$. Assume that $\eta \circ P=0$ for some second order differential operator $P$ with metric principal part. Let $\omega$ be a state over the quasilocal algebra $\mathcal{F}$ which we assume to be quasifree and continuous in the bosonic case. If $\omega$ is a ground-or KMS-state with respect to the automorphism group $\tau_{t}$ induced by the Killing flow $h_{t}$, then $\omega$ has the Reeh-Schlieder property.

We postpone the proof for a moment. Let $\mathcal{O} \subset M$ be a nonvoid relatively compact open set and $\mathcal{B}(\mathcal{O}) \subset \mathcal{F}(\mathcal{O})$ the $*$-subalgebra consisting of those elements $a \in$ $\mathcal{F}(\mathcal{O})$ for which there exists a neighbourhood $I \subset \mathbb{R}$ of 0 , such that $\tau_{I}(a) \subset$ $\mathcal{F}(\mathcal{O})$. Let $\mathcal{O}_{1} \subset M$ be another nonvoid open set such that $\overline{\mathcal{O}_{1}} \subset \mathcal{O}$. We clearly have the inclusions

$$
\mathcal{F}\left(\mathcal{O}_{1}\right) \subset \mathcal{B}(\mathcal{O}) \subset \mathcal{F}(\mathcal{O})
$$

We denote the strongly continuous unitary group, implementing $\tau_{t}$ on the GNSHilbert space, by $\tilde{U}(t)$. One has 
Lemma 4.9. Let $\mathcal{E}:=\overline{\pi_{\omega}(\mathcal{B}(\mathcal{O})) \Omega_{\omega}}$. Then $\mathcal{E}$ is invariant under the action of $\tilde{U}(t)$, i.e. $\tilde{U}(\mathbb{R}) \mathcal{E} \subset \mathcal{E}$.

Proof. Let $\psi \in \mathcal{E}^{\perp}$. For each $a \in \pi_{\omega}(\mathcal{B}(\mathcal{O}))$ we then have at least for some open neighbourhood $I \subset \mathbb{R}$ of 0

$$
f(t):=\left\langle\psi, \tilde{U}(t) a \Omega_{\omega}\right\rangle=0 \quad \forall t \in I .
$$

Since $\omega$ is a KMS-state $(\beta>0)$ or a ground-state $(\beta=\infty), f(t)$ is the boundary value of a function $F(z)$ which is analytic on the strip

$$
\mathcal{D}_{\beta / 2}=\{z \in \mathbb{C} ; 0<\operatorname{Im}(z)<\beta / 2\}
$$

and bounded and continuous on $\overline{\mathcal{D}_{\beta / 2}}$. By the Schwartz reflection principle $f(t)$ vanishes on the whole real axis. Therefore $\left\langle\tilde{U}(t) \psi, a \Omega_{\omega}\right\rangle=0$ for all $t \in \mathbb{R}$. Hence, $\mathcal{E}^{\perp}$ is invariant under the action of $\tilde{U}(t)$.

We are now able to give the proof of the main theorem:

\section{Proof of theorem 4.8}

We denote by $\mathcal{R}$ the von Neumann algebra $\bigvee_{t \in \mathbb{R}} \tilde{U}(t) \pi_{\omega}\left(\mathcal{F}\left(\mathcal{O}_{1}\right)\right) \tilde{U}(t)^{*}$. Lemma 4.9 implies that $\mathcal{E}$ is invariant under the action of $\mathcal{R}$, i.e. $\mathcal{R} \mathcal{E} \subset \mathcal{E}$. By theorem 4.6 $\mathcal{R}=\pi_{\omega}(\mathcal{F})^{\prime \prime}$. Hence $\Omega_{\omega}$ is cyclic for $\mathcal{R}$ and therefore $\mathcal{E}=\mathcal{H}_{\omega}$. By the inclusions (19) we have $\mathcal{E} \subset \overline{\pi_{\omega}(\mathcal{F}(\mathcal{O})) \Omega_{\omega}}$ and hence $\Omega_{\omega}$ is cyclic for $\pi_{\omega}(\mathcal{F}(\mathcal{O})$ ).

\section{Examples of linear field theories}

In this section $(M, g)$ will be an oriented time-oriented 4-dimensional Lorentzian manifold which is globally hyperbolic in the sense that it admits a smooth Cauchy surface. For some subset $\mathcal{O} \subset M$ the set of points, which can be reached by future/past directed causal curves emanating from $\mathcal{O}$, will be denoted by $J^{ \pm}(\mathcal{O})$.

The free Dirac field (see [14]). It is known that $M$ possesses a trivial spin structure given by a $\operatorname{Spin}^{+}(3,1)$-principal bundle $S M$ and a two-fold covering map $S M \rightarrow F M$ onto the bundle $F M$ of oriented time-oriented orthonormal frames. One can now construct the Dirac bundle $D M$ which is associated to $S M$ by the spinor representation and is a natural module for the Clifford algebra bundle 
Cliff( $(T M)$ (see [4] and [28]). Furthermore, the Levi-Civita connection on $T M$ induces a connection on $D M$ with covariant derivative

$$
\nabla: \Gamma(S M) \rightarrow \Gamma\left(S M \otimes T^{*} M\right) .
$$

Given a vector field $n$ we write as usual $h$ for the section in the Clifford algebra bundle $\gamma(n)$, or in local coordinates $\gamma^{i} n_{i}$. There exists an antilinear bijection $\Gamma(D M) \rightarrow \Gamma\left(D M^{*}\right): u \rightarrow u^{+}$, the Dirac conjugation, which in the standard representation in a local orthonormal spin frame has the form $u^{+}=\bar{u} \gamma^{0}$, where the bar denotes complex conjugation in the dual frame. We use the symbol ${ }^{+}$also for the inverse map. Canonically associated with the Dirac bundle there is the Dirac operator which in a frame takes the form

$$
\not \nabla=\gamma^{i} \nabla_{e_{i}} .
$$

The Dirac equation for mass $m \geq 0$ is

$$
(-i \not \nabla+m) u=0, \quad u \in \Gamma(D M) .
$$

The Dirac equation has unique advanced and retarded fundamental solutions $S^{ \pm}: \Gamma_{0}(D M) \rightarrow \Gamma(D M)$ satisfying

$$
\begin{gathered}
(-i \not \nabla+m) S^{ \pm}=S^{ \pm}(-i \not \nabla+m)=\mathrm{id} \quad \text { on } \quad \Gamma_{0}(D M), \\
\operatorname{supp}\left(S^{ \pm} f\right) \subset J^{ \pm}(\operatorname{supp}(f)) .
\end{gathered}
$$

We can define the operator $S:=S^{+}-S^{-}$and form the pre-Hilbert space

$$
H:=\Gamma_{0}(D M) / \operatorname{ker}(S)
$$

with inner product

$$
\left\langle\left[u_{1}\right],\left[u_{2}\right]\right\rangle_{H}:=-i \int_{M}\left(u_{1}^{+}, S u_{2}\right)(x) d \mu(x),
$$

where $(\cdot, \cdot)$ denotes the dual pairing between the fibres of $D M$ and $D M^{*}$ and $\mu(x)$ the canonical (pseudo)-Riemannian measure on $M$. $[f]$ denotes the equivalence class containing $f$. Analogously we form the pre-Hilbert space

$$
H^{+}:=\Gamma_{0}\left(D M^{*}\right) /(\operatorname{ker}(S))^{+}
$$


with inner product

$$
\left\langle\left[v_{1}\right],\left[v_{2}\right]\right\rangle_{H^{+}}:=\left\langle\left[v_{2}^{+}\right],\left[v_{1}^{+}\right]\right\rangle_{H}
$$

We note that $S$ maps $H$ onto the space of smooth solutions to the Dirac equation whose supports have compact intersections with any Cauchy surface. The construction of the Dirac field starts with the Hilbert space $\mathcal{H}:=\overline{H \oplus H^{+}}$and the antiunitary involution

$$
\mathcal{K}: \mathcal{H} \rightarrow \mathcal{H}, \quad u \oplus v \rightarrow v^{+} \oplus u^{+}
$$

By construction we have a map

$$
\eta: \Gamma_{0}\left(D M \oplus D M^{*}\right) \rightarrow \mathcal{H} ; \quad f \oplus g \rightarrow[f] \oplus[g]
$$

with dense range. Let $G$ be the identity component of the group of isometries of $M$ and $\tilde{G}$ its universal covering group. $\tilde{G}$ acts canonically on $F M$ and since $\tilde{G}$ is simply connected this actions lifts uniquely to a smooth action on $S M$, yielding a smooth equivariant action on the bundles $D M$ and $D M^{*}$, making them $\tilde{G}$-vector bundles. The Dirac operator $\not \nabla$ and its conjugate $\not^{+}:={ }^{+} \circ \not \circ^{+}$are both invariant under these actions and hence the representation of $\tilde{G}$ on $\Gamma_{0}\left(D M \oplus D M^{*}\right)$ gives rise to a unitary representation $\rho$ of $\tilde{G}$ on $\mathcal{H}$. Taking $E:=D M \oplus D M^{*}$ one shows that $(\mathcal{H}, \mathcal{K}, E, \eta, \rho)$ is a linear fermionic field theory on $M$. Moreover, the differential operator $P=\not \nabla \oplus \not^{+}: \Gamma_{0}(E) \rightarrow \Gamma_{0}(E)$ maps to the kernel of $\eta$, i.e. $\eta \circ P=0$. The square of $P$ has metric principal part.

The real scalar field (see [13]). The construction of the Klein-Gordon field starts with the Klein-Gordon operator for mass $m \geq 0$ :

$$
P:=\square_{g}+m^{2}
$$

where $\square_{g}=g^{i k} \nabla_{i} \nabla_{k}$ and $\nabla$ is the Levi-Civita covariant derivative. This operator acts on the real-valued smooth functions with compact support $C_{0 r}^{\infty}(M)$. It has unique advanced and retarded fundamental solutions $F_{s}^{ \pm}: C_{0 r}^{\infty}(M) \rightarrow C_{r}^{\infty}(M)$ satisfying

$$
\begin{gathered}
P F_{s}^{ \pm}=F_{s}^{ \pm} P=\text { id on } C_{0 r}^{\infty}(M), \\
\operatorname{supp}\left(F_{s}^{ \pm} f\right) \subset J^{ \pm}(\operatorname{supp}(f)) .
\end{gathered}
$$


With $F_{s}:=F_{s}^{+}-F_{s}^{-}, \hat{\sigma}\left(f_{1}, f_{2}\right):=\int_{M} f_{1} F_{s}\left(f_{2}\right) w$ defines an antisymmetric bilinear form on $C_{0 r}^{\infty}(M) \times C_{0 r}^{\infty}(M)$, where $w$ is the pseudo-Riemannian volume form on $M$. Defining $\mathcal{W}:=C_{0 r}^{\infty}(M) / \operatorname{ker}\left(F_{s}\right)$ with quotient map $\eta$, the bilinear form $\sigma\left(\eta\left(f_{1}\right), \eta\left(f_{2}\right)\right):=\hat{\sigma}\left(f_{1}, f_{2}\right)$ on $\mathcal{W}$ is symplectic. We have a canonical linear action of the group $G$ on $C_{0 r}^{\infty}(M)$ which leaves $\operatorname{ker}\left(F_{s}\right)$ and $\hat{\sigma}$ invariant and hence gives a representation $\rho$ of $G$ on $\mathcal{W}$ by symplectomorphisms. Taking the trivial bundle $M \times \mathbb{R}$ for $E$, one shows that $(\mathcal{W}, \sigma, E, \eta, \rho)$ is a linear bosonic field theory on $M$ and $\eta \circ P=0$. Note that $P$ has metric principal part.

Remark 5.1. Since the complex scalar field consists of two independent real scalar fields it fits into this framework as well.

The Proca field (see [16]). Let $d$ be the exterior derivative of differential forms, * the Hodge star operator and $\delta=* d *$. Take the cotangent bundle for $E$. For mass $m>0$ the Proca equation for sections $f \in \Gamma_{0}(E)$ is

$$
\left(\delta \circ d+m^{2}\right) f=0,
$$

which is equivalent to the hyperbolic system

$$
\begin{gathered}
\left(\square_{g}+m^{2}\right) f=\left(\delta \circ d+d \circ \delta+m^{2}\right) f=0, \\
\delta f=0 .
\end{gathered}
$$

We define the differential operator $\tilde{P}: \Gamma_{0}(E) \rightarrow \Gamma_{0}(E)$ by

$$
\tilde{P}:=\square_{g}+m^{2} .
$$

It has unique advanced and retarded fundamental solutions $\tilde{F}_{p}^{ \pm}: \Gamma_{0}(E) \rightarrow \Gamma(E)$ satisfying

$$
\begin{gathered}
\tilde{P} \tilde{F}_{p}^{ \pm}=\tilde{F}_{p}^{ \pm} \tilde{P}=\mathrm{id} \quad \text { on } \quad \Gamma_{0}(E), \\
\operatorname{supp}\left(\tilde{F}_{p}^{ \pm} f\right) \subset J^{ \pm}(\operatorname{supp}(f)) .
\end{gathered}
$$

We define the operators $F_{p}^{ \pm}:=\left(m^{-2} d \circ \delta+1\right) \tilde{F}_{p}^{ \pm}$. It is not difficult to see that the $F_{p}^{ \pm}$are the unique fundamental solutions for the operator $P=\delta \circ d+m^{2}$ with the above properties. With $F_{p}:=F_{p}^{+}-F_{p}^{-}, \hat{\sigma}\left(f_{1}, f_{2}\right):=\int_{M} f_{1} \wedge * F_{p}\left(f_{2}\right)$ defines an antisymmetric bilinear form on $\Gamma_{0}(E) \times \Gamma_{0}(E)$. Taking $\mathcal{W}:=\Gamma_{0}(E) / \operatorname{ker}\left(F_{p}\right)$ 
with quotient map $\eta$, the bilinear form $\sigma\left(\eta\left(f_{1}\right), \eta\left(f_{2}\right)\right):=\hat{\sigma}\left(f_{1}, f_{2}\right)$ on $\mathcal{W}$ is symplectic. The pullback of forms induces a linear action of $G$ on $\Gamma_{0}(E)$ which leaves $\operatorname{ker}\left(F_{p}\right)$ and $\hat{\sigma}$ invariant and hence gives rise to a representation $\rho$ of $G$ on $\mathcal{W}$ by symplectomorphisms. Again one can show that $(\mathcal{W}, \sigma, E, \eta, \rho)$ is a linear bosonic field theory and moreover, $\eta \circ \tilde{P}=\eta \circ P=0$.

One gets the following corollary.

Corollary 5.2. Let $\left(M, g, h_{t}\right)$ be a connected stationary globally hyperbolic oriented time-oriented 4-dimensional Lorentzian manifold. Let $\mathcal{O} \rightarrow \mathcal{F}(\mathcal{O})$ be a net of field algebras for one of the following free fields:

- The real or complex scalar field for mass $m \geq 0$,

- The Proca field for mass $m>0$,

- The Dirac field for mass $m \geq 0$,

Assume that $\omega$ is a state over the field algebra $\mathcal{F}$ which we require to be quasifree and continuous in the bosonic case and which is a ground-or KMS-state with respect to the canonical time translations. Then $w$ has the Reeh-Schlieder property.

\section{Acknowledgements}

The author would like to thank Prof. M. Wollenberg and Dr. R. Verch for useful discussions and comments. This work was supported by the Deutsche Forschungsgemeinschaft within the scope of the postgraduate scholarship programme "Graduiertenkolleg Quantenfeldtheorie" at the University of Leipzig.

\section{References}

[1] H. Araki. On Quasifree States of CAR and Bogoliubov Automorphisms. Publ. RIMS Kyoto Univ., 6:385-442, 1970/71.

[2] N. Aroszajn. A unique continuation theorem for solutions of elliptic partial differential equations or inequalities of second order. J. Math. Pures Appl., 36:235-249, 1957. 
[3] C. Bär. Localization and semibounded energy - a weak unique continuation theorem. math-ph/9910023.

[4] H. Baum. Spin-Strukturen und Dirac-Operatoren über pseudoriemannschen Mannigfaltigkeiten. Teubner, Leipzig, 1981.

[5] H.J. Borchers. On revolutionizing quantum field theory. lqp-preprint 99042900 , to appear in J. Math. Phys.

[6] H.J. Borchers. Über die Vollständigkeit lorentzinvarianter Felder in einer zeitartigen Röhre. Nuovo Cim., 19:787, 1961.

[7] O. Bratteli and D.W. Robinson. Operator Algebras and Quantum Statistical Mechanics 2. Springer, 1996.

[8] R. Brunetti, K. Fredenhagen, and M. Kohler. The microlocal spectrum condition and wick polynomials of free fields on curved space-times. Commun. Math. Phys., 180:633-652, 1996.

[9] D. Buchholz, O. Dreyer, M. Florig, and S. J. Summers. Geometric modular action and space-time symmetry groups. math-ph/9805026, to appear in Rev. Math. Phys.

[10] N. Dencker. On the propagation of polarization sets for systems of real principle type. J. Funct. Anal., 46:351-372, 1982.

[11] J. Dieudonne. Grundzüge der modernen Analysis, volume 3. Vieweg Verlag, Braunschweig, 1976.

[12] J. Dieudonne. Grundzüge der modernen Analysis, volume 7. Vieweg Verlag, Braunschweig, 1976.

[13] J. Dimock. Algebras of local observables on a manifold. Commun. Math. Phys., 77:219-228, 1980.

[14] J. Dimock. Dirac quantum fields on a manifold. Trans. Am. Math. Soc., 269:133-147, 1982. 
[15] S. A. Fulling. Aspects of Quantum Field Theory in Curved Space-Time. Univ. Pr., Cambridge, UK, 1989.

[16] E. P. Furlani. Quantization of massive vector fields in curved space- time. $J$. Math. Phys., 40:2611, 1999.

[17] I.M. Gelfand and N.J. Wilenkin. Verallgemeinerte Funktionen. Deutscher Verlag der Wissenschaften, Berlin, 1964.

[18] D. Guido, R. Longo, J. E. Roberts, and R. Verch. Charged sectors, spin and statistics in quantum field theory on curved space-times. 1999. mathph/9906019, to appear in Rev. Math. Phys.

[19] R. Haag. Local quantum physics: Fields, particles, algebras. Springer, Berlin, Germany, 1992.

[20] L. Hörmander. The Analysis of Linear Partial Differential Operators III. Springer, 1985.

[21] L. Hörmander. The Analysis of Linear Partial Differential Operators I. Springer, 1990.

[22] C. D. Jäkel. The Reeh-Schlieder property for thermal field theories. hepth/9904049.

[23] H. Jarchow. Locally convex spaces. B. G. Teubner, Stuttgart, 1981.

[24] B. S. Kay. Linear spin-zero quantum fields in external gravitational and scalar fields. i. a one particle structure for the stationary case. Commun. Math. Phys., 62:55, 1978.

[25] B. S. Kay. The double wedge algebra for quantum fields on schwarzschild and minkowski space-times. Commun. Math. Phys., 100:57, 1985.

[26] B. S. Kay. Sufficient conditions for quasifree states and an improved uniqueness theorem for quantum fields on space-times with horizons. J. Math. Phys., 34:4519-4539, 1993. 
[27] B. S. Kay and R. M. Wald. Theorems on the uniqueness and thermal properties of stationary, nonsingular, quasifree states on space-times with a bifurcate killing horizon. Phys. Rept., 207:49-136, 1991.

[28] H. B. Lawson and M. L. Michelsohn. Spin Geometry. Princeton University press, 1989.

[29] K. Maurin. General Eigenfunction Expansions and Unitary Representations of Topological Groups. Polish Scientific Publishers, Warszawa, 1968.

[30] B. O’Neill. Semi-Riemannian Geometry. Academic Press, 1983.

[31] A. Pietsch. Nukleare Lokalkonvexe Räume. Akademie-Verlag, Berlin, 1965.

[32] M. J. Radzikowski. The Hadamard condition and Kay's conjecture in (axiomatic) quantum field theory on curved space-timer. PhD-Thesis, 1992.

[33] H. Reeh and S.Schlieder. Bermerkungen zur Unitäräquivalenz von Lorentzinvarianten Feldern. Nuovo Cimento, 22:1051-1068, 1961.

[34] H. Sahlmann and R. Verch. Passivity and microlocal spectrum condition. math-ph/0002021.

[35] A. Strohmaier. The Reeh-Schlieder property for the Dirac field on static spacetimes. math-ph/9911023.

[36] R. Verch. Antilocality and a Reeh-Schlieder theorem on manifolds. Lett. Math. Phys., 28:143-154, 1993.

[37] R. M. Wald. The back reaction effect in particle creation in curved spacetime. Commun. Math. Phys., 54:1, 1977.

[38] R. M. Wald. Quantum Field Theory in Curved Spacetime and Black Hole Thermodynamics. Chicago Lectures in Physics, 1994. 